\title{
Improved Multi-Channel Ranging Precision Bound for Narrowband LPWAN in Multipath Scenarios
}

\author{
Florian Wolf* ${ }^{\dagger}$, Christophe Villien*, Sébastien de Rivaz*, François Dehmas* and Jean-Pierre Cances ${ }^{\dagger}$ \\ * CEA-Leti Minatec Campus, 17 rue des Martyrs, 38054 Grenoble Cedex 09, France \\ Email: \{florian.wolf, christophe.villien, sebastien.derivaz, francois.dehmas\}@cea.fr \\ † Université de Limoges, CNRS, XLIM, UMR 7252, 87000 Limoges, France \\ Email: \{florian.wolf, jean-pierre.cances\}@xlim.fr
}

\begin{abstract}
Accurate range estimation with narrowband low power radio devices is challenging due to limited signal bandwidth and high frequency offsets of low-cost oscillators. The present paper provides new results concerning the Cramer Rao bounds of narrowband ranging systems applying a multi-channel coherent processing approach. Particularly, compared to existing literature, oscillator frequency offsets and multipath influence are taken into account. By numerical simulation it is shown, how the radio channel frequency selectivity degrades the ranging precision. Finally, a few guidelines for the design of new long range low-cost ranging systems are provided.
\end{abstract}

Index Terms-CRLB, LPWAN localization, range estimation, phase of arrival, channel estimation

\section{INTRODUCTION}

Long range low power radio devices are designed for wireless sensor network (WSN) applications, where low-cost devices with high energy constraints exchange data over several kilometers within a network [1]. Industrial scientific and medical (ISM) band sub-GHz radio transceivers [2]-[5] for so called low power wide area networks (LPWAN) achieve these requirements at the trade-off of very limited transmission rates and hence with narrow radio signal bandwidths.

In many use-cases, position information of the radio devices is an asset to the transmitted data or can even be used for LPWA network management [6]. The use of GNSS systems is often excluded for cost or power consumption reasons, or signal unavailability in scenarios, where the device to satellite lineof-sight is obstructed. Obtaining GNSS accuracy equivalent position estimation from radio signals emitted by the devices, for data transmission, is in the focus of current research. Position estimation is a two-step process. First, metrics such as time of arrival (ToA) or angle of arrival (AoA) are extracted from radio signals, then, in a second step, these metrics are combined to estimate position [7].

ToA propagation delay measurement methods are widely deployed in ultra-wideband (UWB) systems, where large instantaneous bandwidths offer excellent range resolution and accuracy [8]. However, ToA is challenging in narrowband systems as precision is inverse proportional to bandwidth [9].

In order to overcome the lack of large instantaneous bandwidths, techniques such as frequency modulated continuous wave (FMCW) and stepped frequency (SF) radar [10] are utilized to increase bandwidth sequentially. In contrast to UWB techniques, where the channel impulse response is measured in time domain, FMCW and SF radars estimate the channel transfer function in frequency domain. Phase coherent processing schemes for these techniques are studied in the multi-channel context for RFID [11], WiFi [12] and Zigbee [13], [14] active device localization. In all these contributions, an accuracy gain over single channel ToA estimation has been demonstrated. The aforementioned, short to medium range transceivers use instantaneous bandwidths of a few MHz, compared to LPWAN radio devices, where signal bandwidths range from a few hundred $\mathrm{Hz}$ to a few hundred $\mathrm{kHz}$. The long transmission times herein involved, suggest that sampling and carrier frequency offsets (SFO, CFO), and radio channel frequency selectivity will be a major issue for ranging accuracy.

For the prediction of maximum attainable range estimation accuracy, the Cramer Rao lower bound (CRLB) from estimation theory [15] is used. Multi-carrier ranging CRLBs have been derived analytically for multipath channels with interference [16]. The focus is on optimal subcarrier allocation in interference scenarios for OFDM systems. Hardware imperfections such as $\mathrm{CFO}$ are not considered and simulation results are given for wideband transmissions. Furthermore, the CRLB is pessimistic as it considers strong, unknown multipath. Hence it does not show the possible precision gain [14] of multi-channel ranging in weak multipath over the approach of averaging independent single channel measurements.

In order to give more detailed CRLBs, the main contributions of this paper are:

- Extension of the signal model from [13], [14] to include multipath. It serves as detailed signal model for two-way and differential delay estimation approaches.

- By theoretical considerations and numerical simulation, it is shown, that CFO has no impact on delay estimation for two-way ranging.

- Numerical results for two-way narrowband LPWAN ranging precision bounds are given for typical ETSI channel models. These detailed bounds are lower than the pessimistic CRLBs from literature [16].

This paper is organized as follows. Section II describes the signal model for a generic transceiver hardware architecture. Section III introduces the CRLB, radio channel representation and CRLBs from literature. Numerical simulation results and further prospects are given in Section IV and Section V. 


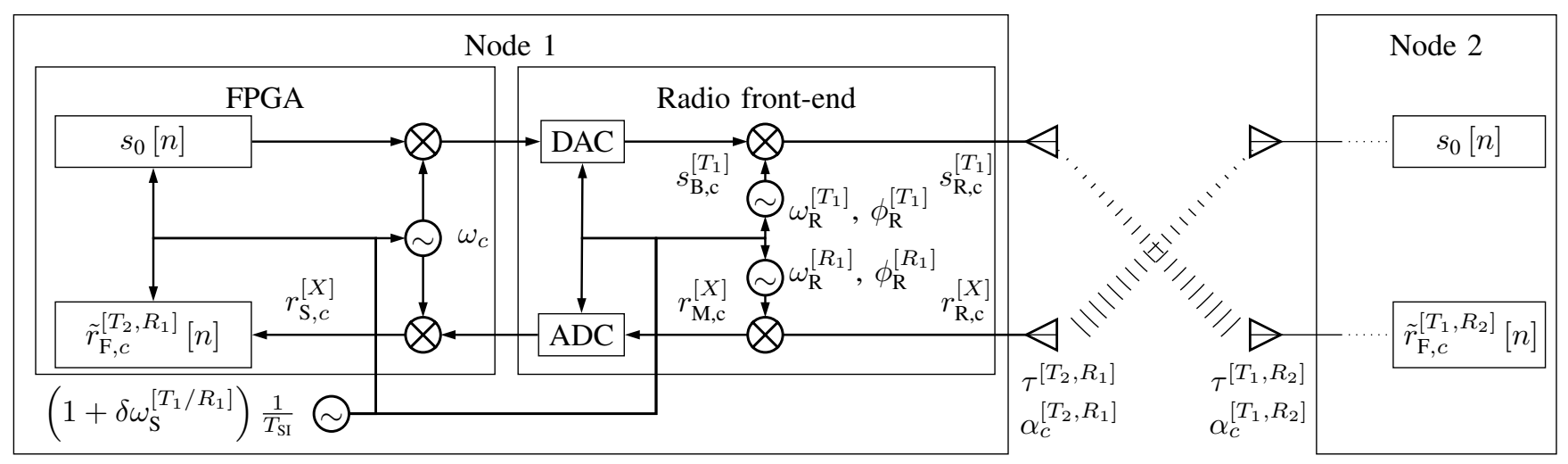

Fig. 1. Generic hardware architecture for transmitter and receiver.

\section{Signal Model}

Two-way delay estimation is a ranging handshake protocol for unsynchronized nodes [9]. The signal model from [13], [14] is adapted for two-way ranging with a transceiver hardware architecture depicted in Fig. 1 and multipath propagation. The baseband signal will be up- and down-converted in a digital step to intermediate frequency (IF) $\omega_{c}$ to maintain phase coherence between transmission and reception, and by analogue mixing to fixed radio frequency $\omega_{\mathrm{RI}}$. Details are explained hereafter.

The baseband waveform $s_{0}[n]=s_{0}\left(n T_{\mathrm{SI}}\right)$ with $n \in\left[0, N_{\mathrm{S}}-1\right]$, number of samples $N_{\mathrm{S}}$ and sampling frequency $1 / T_{\mathrm{SI}}$, is known to transmitter $T$ and receiver $R$. The local time scale of transmitter $T$ is given by

$$
t^{[T]}=\left(1+\delta \omega_{\mathrm{S}}^{[T]}\right) t+t_{0}^{[T]}
$$

with global time $t$, normalized sampling clock frequency offset $\delta \omega_{\mathrm{S}}^{[T]}$ and time offset $t_{0}^{[T]}$. The baseband waveform is numerically up-converted to the channel frequency $\omega_{c}$. On global time base and for ideal digital-to-analogue conversion (DAC), it is given by

$$
s_{\mathrm{B}, \mathbf{c}}^{[T]}(t)=s_{0}\left(t^{[T]}\right) e^{j \omega_{c} t^{[T]}} .
$$

It has to be noted, that no phase shift term is added in upconversion since this is a pure numerical process.

According to Fig. 1, timing reference for sampling and the local oscillator are the same, so that the radio frequency (RF) is equal to $\omega_{\mathrm{R}}^{[T]}=\left(1+\delta \omega_{\mathrm{S}}^{[T]}\right) \omega_{\mathrm{RI}}$, with $\omega_{\mathrm{RI}}$ ideal RF. The up-converted radio signal is denoted

$$
s_{\mathrm{R}, c}^{[T]}(t)=s_{\mathrm{B}, c}^{[T]}(t) e^{j\left(\omega_{\mathrm{R}}^{[T]} t+\phi_{\mathrm{R}}^{[T]}\right)},
$$

where the phase shift $\phi_{\mathrm{R}}^{[T]}$ accounts for unknown phase of the transmit local oscillator.

The RF signal is transmitted over the wireless radio channel and received by receiver $R$. Under the assumption of a narrowband signal $s_{0}(t)$, the transmitter and receiver complex radio front end gains and the complex radio channel coefficient at frequency $\left(\omega_{\mathrm{RI}}+\omega_{c}\right)\left(1+\delta \omega_{\mathrm{S}}^{[T]}\right)$ can be summarized as $\alpha_{c}^{[X]}$ with $[X]=[T, R]$. The signal at the mixer input on the receiver side $^{1}$ is given by

$$
r_{\mathrm{R}, c}^{[X]}(t)=\alpha_{c}^{[X]} s_{\mathrm{R}, c}^{[T]}\left(t-\tau^{[X]}\right) .
$$

The propagation delay between transmitter $T$ and receiver $R$ is denoted $\tau^{[X]}=d^{[X]} / c_{0}$ with transmitter-receiver distance $d^{[X]}$ and radio wave propagation velocity $c_{0}$. After down-conversion, the analog IF signal is given by

$$
r_{\mathrm{M}, c}^{[X]}(t)=r_{\mathrm{R}, c}^{[X]}(t) e^{-j\left(\omega_{\mathrm{R}}^{[R]} t+\phi_{\mathrm{R}}^{[R]}\right)},
$$

with $\omega_{\mathrm{R}}^{[R]}=\left(1+\delta \omega_{\mathrm{S}}^{[R]}\right) \omega_{\mathrm{RI}}$ and unknown phase $\phi_{\mathrm{R}}^{[R]}$. The receiver time reference is given by

$$
t^{[R]}=\left(1+\delta \omega_{\mathrm{S}}^{[R]}\right) t+t_{0}^{[R]} .
$$

After numerical down-conversion, this yields the digital baseband signal

$$
r_{\mathrm{S}, c}^{[X]}\left(t^{[R]}\right)=r_{\mathrm{M}, c}^{[X]}\left(\frac{t^{[R]}-t_{0}^{[R]}}{1+\delta \omega_{\mathrm{S}}^{[R]}}\right) e^{-j \omega_{c} t^{[R]}} .
$$

The following notation is used, in order to write the sampled (ADC) observation in vector form ${ }^{2}$

$$
\begin{aligned}
\boldsymbol{r}_{\mathrm{F}}^{[X]}= & {\left[\operatorname{Re}\left\{r_{\mathrm{F}, 0}^{[X]}\right\} \operatorname{Im}\left\{r_{\mathrm{F}, 0}^{[X]}\right\} \ldots\right.} \\
& \left.\operatorname{Re}\left\{r_{\mathrm{F}, C-1}^{[X]}\right\} \operatorname{Im}\left\{r_{\mathrm{F}, C-1}^{[X]}\right\}\right]^{\mathrm{T}} \in \mathbb{R}^{2 C \times 1},
\end{aligned}
$$

where

$$
r_{\mathrm{F}, c}^{[X]}[n]=r_{\mathrm{S}, c}^{[X]}\left(\left(n+l_{c}^{[X]} N_{\mathrm{S}}\right) T_{\mathrm{SI}}\right),
$$

with transmission slot $l_{c}^{[X]}$ specified hereafter.

Coarse time synchronization ${ }^{3}$ between transmitter $T$ and receiver $R$ and sufficient zero samples at the beginning and end of the waveform $s_{0}[n]$ ensure robustness to SFO. For $C$ successive transmissions on the channel frequencies $\boldsymbol{\omega}_{c}=\left[0, \Delta \omega_{c}, 2 \Delta \omega_{c}, \ldots,(C-1) \Delta \omega_{c}\right]^{\mathrm{T}}$, the received signal can be expressed as function of the baseband waveform $s_{0}(t)$.

\footnotetext{
${ }^{1}$ Noise is added after down conversion, see (10).

${ }^{2} \operatorname{Re}\{\cdot\}$ and $\operatorname{Im}\{\cdot\}$ are real and imaginary part respectively.

${ }^{3} \mathrm{Can}$ be assumed as given by a single channel ToA-based method.
} 
In summary, the received signal on channel $c$ yields

$$
\begin{aligned}
\tilde{r}_{\mathrm{F}, c}^{[X]}[n] & =r_{\mathrm{F}, c}^{[X]}[n]+n_{c}^{[X]}[n] \\
& =\alpha_{c}^{[X]} s_{0}\left(A_{c}^{[X]}\right) e^{j B_{c}^{[X]}}+n_{c}^{[X]}[n],
\end{aligned}
$$

with $\alpha_{c}^{[X]}$ (channel coefficient), $A_{c}^{[X]}=T_{\mathrm{F}}^{[X]}\left(n-n_{\mathrm{F}, c}^{[X]}\right)$ (time dilatation, time offset, propagation delay) and $B_{c}^{[X]}=\left(k_{\mathrm{F}, c}^{[X]} n+\phi_{\mathrm{F}, c}^{[X]}\right) \quad(\mathrm{CFO}$, phase offset $)$ detailed in (11) to (16). Complex additive white Gaussian transmitter, channel and receiver noise is denoted $n_{c}^{[X]}[n]$. Its in phase and quadrature variances are given by $\sigma_{\{\operatorname{Re}, \operatorname{Im}\}}^{2}=\frac{1}{2 S N R}$, where $S N R$ denotes signal-to-noise ratio. Symbol to noise power density ratio is given by $\frac{E_{\mathrm{s}}}{N_{0}}=S N R \cdot N_{\mathrm{S}}$.

In the following, this general signal model is simplified for two-way ranging between node $N_{1}$ and $N_{2}$, transmitting in the transmission slots $l_{c}^{[X]}=2 c$ and $l_{c}^{[X]}=2 c+1$ respectively. As depicted in Fig. 1, clock reference for transmit and receive path are common $\left(\delta \omega_{\mathrm{S}}^{\left[T_{x}\right]}=\delta \omega_{\mathrm{S}}^{\left[R_{x}\right]}, t_{0}^{\left[T_{x}\right]}=t_{0}^{\left[R_{x}\right]}\right)$. Node $N_{1}$ is assigned the reference node, hence its time and frequency offsets are set to zero $\left(t_{0}^{\left[T_{1}\right]}=t_{0}^{\left[R_{1}\right]}=0, \delta \omega_{\mathrm{S}}^{\left[T_{1}\right]}=\delta \omega_{\mathrm{S}}^{\left[R_{1}\right]}=0\right)$. Frequency offsets and the radio channel are considered time invariant over the total signaling duration. CFO impact on channel coefficients is neglected and the radio channel is assumed symmetric (11), (12).

Remaining parameters for (10) are given as follows ${ }^{4}$

$$
\begin{aligned}
a_{c} e^{j \phi_{c}}= & \alpha_{c}^{\left[T_{1}, R_{2}\right]} \approx \alpha_{c}^{\left[T_{2}, R_{1}\right]}, \\
\tau= & \tau^{\left[T_{1}, R_{2}\right]}=\tau^{\left[T_{2}, R_{1}\right]}, \\
T_{\mathrm{F}}^{[X]}= & T_{\mathrm{SI}}\left\{\begin{array}{l}
\frac{1}{1+\delta \omega_{\mathrm{S}}} \\
1+\delta \omega_{\mathrm{S}},
\end{array}\right. \\
n_{\mathrm{F}, c}^{[X]}= & \frac{1}{T_{\mathrm{SI}}}\left\{\begin{array}{l}
{\left[t_{0}+\left(1+\delta \omega_{\mathrm{S}}\right) \tau+\delta \omega_{\mathrm{S}} l_{c}^{[X]} N_{\mathrm{S}} T_{\mathrm{SI}}\right]} \\
\frac{1}{\left(1+\delta \omega_{\mathrm{S}}\right)}\left[-t_{0}+\left(1+\delta \omega_{\mathrm{S}}\right) \tau-\delta \omega_{\mathrm{S}} l_{c}^{[X]} N_{\mathrm{S}} T_{\mathrm{SI}}\right]
\end{array}\right. \\
k_{\mathrm{F}, c}^{[X]}= & T_{\mathrm{SI}}\left(\omega_{c}+\omega_{\mathrm{RI}}\right)\left\{\begin{array}{l}
-\frac{\delta \omega_{\mathrm{S}}}{1+\delta \omega_{\mathrm{S}}}, \\
\delta \omega_{\mathrm{S}},
\end{array}\right. \\
\phi_{\mathrm{F}, c}^{[X]}= & k_{\mathrm{F}, c}^{[X]} l_{c}^{[X]} N_{\mathrm{S}} \\
& +\left\{\begin{array}{l}
\frac{-\omega_{c}+\omega_{\mathrm{RI}} \delta \omega_{\mathrm{S}}}{1+\delta \omega_{\mathrm{S}}} t_{0}-\left(\omega_{c}+\omega_{\mathrm{RI}}\right) \tau+\phi_{\mathrm{R}}^{\left[T_{1}\right]}-\phi_{\mathrm{R}}^{\left[R_{2}\right]} \\
\omega_{c} t_{0}-\left(\omega_{c}+\omega_{\mathrm{RI}}\right)\left(1+\delta \omega_{\mathrm{S}}\right) \tau+\phi_{\mathrm{R}}^{\left[T_{2}\right]}-\phi_{\mathrm{R}}^{\left[R_{1}\right]}
\end{array}\right.
\end{aligned}
$$

\section{CRAMER RAO LOWER BOUNDS}

Increasing estimation precision of the propagation delay $\tau$ is the main objective of delay based ranging methods. Precision is in general degraded by noise and other unknown parameters (e.g. CFO, radio channel) which need to be included for a comprehensive model of a real system. Based on the introduced signal model, steps to the CRLB expression for delay and unknown auxiliary parameters is given hereafter. Estimation problem formulation is followed by analytical CRLBs from literature which are used for comparison.

\footnotetext{
${ }^{4}$ Upper choice if $[X]=\left[T_{1}, R_{2}\right]$, lower choice if $[X]=\left[T_{2}, R_{1}\right]$.
}

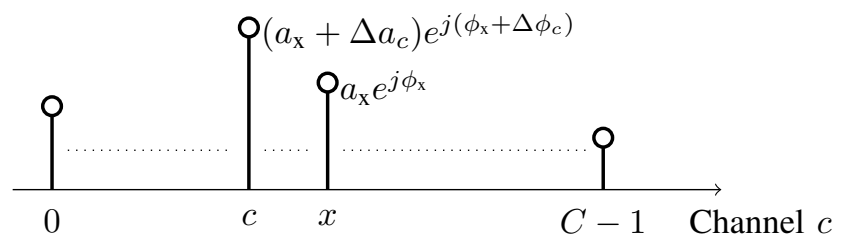

Fig. 2. Channel coefficients for frequency selective radio channels.

\section{A. CRLB for multi-channel ranging in multipath}

The two-way observation is given by

$$
\boldsymbol{R}[n]=\left[\left(\boldsymbol{r}_{\mathrm{F}}^{\left[T_{1}, R_{2}\right]}[n]\right)^{\mathrm{T}}\left(\boldsymbol{r}_{\mathrm{F}}^{\left[T_{2}, R_{1}\right]}[n]\right)^{\mathrm{T}}\right]^{\mathrm{T}} \in \mathbb{R}^{4 C \times 1}
$$

The observation $\boldsymbol{R}$ depends on system parameters $\left\{N_{\mathrm{S}}, C, T_{\mathrm{SI}}, \omega_{\boldsymbol{c}}, \omega_{\mathrm{RI}}, s_{0}(t)\right\}$ and the unknown estimation parameter

$$
\boldsymbol{\theta}=\left[\begin{array}{lll}
\tau & \boldsymbol{A} \boldsymbol{\Phi} \delta \omega_{\mathrm{S}} t_{0} \Delta \phi_{\mathrm{R}}
\end{array}\right]^{\mathrm{T}} \in \mathbb{R}^{(2 C+4) \times 1},
$$

with $\boldsymbol{A}=\left[\Delta a_{0} \ldots \Delta a_{x-1} a_{\mathrm{x}} \Delta a_{x+1} \ldots \Delta a_{C-1}\right] \quad$ and $\boldsymbol{\Phi}=\left[\Delta \phi_{0} \ldots \Delta \phi_{x-1} \phi_{\mathrm{x}} \Delta \phi_{x+1} \ldots \Delta \phi_{C-1}\right] . \quad$ Channel coefficients are represented as amplitude and phase differences to a central channel $x=\left\lceil\frac{C}{2}\right\rceil-1$ as depicted in Fig. 2 and are defined as follows

$$
\begin{aligned}
a_{\mathrm{x}}+\Delta a_{c} & \triangleq a_{c}, \\
\phi_{\mathrm{x}}+\Delta \phi_{c} & \triangleq \phi_{c}+\left(\phi_{\mathrm{R}}^{\left[T_{1}\right]}-\phi_{\mathrm{R}}^{\left[R_{2}\right]}\right), \\
& \Delta \phi_{\mathrm{R}} \triangleq \phi_{\mathrm{R}}^{\left[T_{2}\right]}-\phi_{\mathrm{R}}^{\left[R_{1}\right]}-\phi_{\mathrm{R}}^{\left[T_{1}\right]}+\phi_{\mathrm{R}}^{\left[R_{2}\right]} .
\end{aligned}
$$

The Cramer-Rao lower bound on $\boldsymbol{\theta}$ is given by

$$
\operatorname{Var}(\hat{\boldsymbol{\theta}}) \geq \operatorname{trace}\left(\boldsymbol{I}^{-1}(\boldsymbol{\theta})\right),
$$

with Fisher information matrix (FIM)

$$
\boldsymbol{I}(\boldsymbol{\theta})=\sum_{n=0}^{N_{\mathrm{S}}-1} \boldsymbol{I}_{n}(\boldsymbol{\theta})=\sum_{n=0}^{N_{\mathrm{S}}-1} \boldsymbol{J}_{\boldsymbol{R}, n}^{\mathrm{T}} \boldsymbol{\Sigma}_{\boldsymbol{R}}^{-1} \boldsymbol{J}_{\boldsymbol{R}, n} .
$$

The sample specific Jacobian matrix is $\boldsymbol{J}_{\boldsymbol{R}, n} \in \mathbb{R}^{4 C \times(2 C+4)}$ (see Appendix) and observation covariance matrix is $\quad \boldsymbol{\Sigma}_{\boldsymbol{R}}=\sigma_{\{\mathrm{Re}, \operatorname{Im}\}}^{2} \mathbf{1} \in \mathbb{R}^{4 C \times 4 C}, \quad$ with identity matrix $\mathbf{1} \in \mathbb{R}^{4 C \times 4 C}$. Measurement independence allows to sum over the sample specific FIM $\boldsymbol{I}_{n}(\boldsymbol{\theta}) \in \mathbb{R}^{(2 C+4) \times(2 C+4)}$.

\section{B. Analytical CRLB from literature}

The multi-carrier CRLB for delay estimation from [16, equation 30] is given by ${ }^{5}$

$$
\left(\sigma_{\widehat{\tau}, \mathrm{CRLB}_{[16]}}\right)^{2}=\left(\sum_{k=0}^{K-1} 4 \pi^{2} \frac{E_{\mathrm{s}}}{N_{0}}\left|\alpha_{k}\right|^{2} B W_{\mathrm{RMS}}^{2}\right)^{-1},
$$

with root-mean-squared bandwidth

$$
B W_{\mathrm{RMS}}^{2}=\int_{-\infty}^{\infty} f^{2}\left|S_{0}(f)\right|^{2} d f / \int_{-\infty}^{\infty}\left|S_{0}(f)\right|^{2} d f,
$$

${ }^{5}$ Power weighting, interference and spectrum skewness are omitted. 
where $S_{0}(f)$ represents the spectrum of the waveform $s_{0}(t)$.

Three practical bounds can be derived from (24)

- Single channel bound: For $K=1$ it coincides with the CRLB given in [8], [9]. Precision depends on the waveform bandwidth $B W_{\mathrm{RMS}}=B W_{\mathrm{sym}}$.

- Non-coherent multi-channel bound: Assumes frequency flat fading on each channel $k$ and unknown, independent channel coefficients $\alpha_{k}$. It improves by $1 / C$ with $C=K$ over the single channel bound, which is equivalent to averaging $C$ independent single channel measurements.

- Coherent multi-channel bound: For FMWC and SF radar systems, the CRLB is proportional to $B W_{\mathrm{RMS}}=B W_{\mathrm{coh}}=C \omega_{c} /(2 \pi)[10]$, with $K=1$. Performances are no longer bound by the waveform bandwidth $B W_{\text {sym }}$, but coherent multi-channel bandwidth $B W_{\text {coh }}$ can be freely chosen as a system design parameter. Hence, it is a promising approach for narrowband LPWAN localization.

It is expected, that in multipath scenarios this latter bound cannot be reached due to partial incoherence between the multichannel measurements.

\section{PERformance Bound EVALUATION}

\section{A. Simulation setup and system parameters}

The CRLB for $\boldsymbol{\theta}$ is evaluated numerically since the derivation of an analytical CRLB based on (17) is very complex. Furthermore, a main contribution of the present paper w.r.t. (24) is to add prior information to the channel coefficients. Indeed, assuming their independence for small channel frequency spacing $\omega_{c}$ is pessimistic [16]. The improved CRLB (compare (22)) with prior information is given by [15]

$$
\operatorname{Var}(\hat{\boldsymbol{\theta}}) \geq \operatorname{trace}\left(\left(\boldsymbol{I}(\boldsymbol{\theta})+\boldsymbol{\Sigma}_{\boldsymbol{\theta}}{ }^{-1}\right)^{-1}\right),
$$

where the prior information is given by the estimation parameter $\boldsymbol{\theta}$ covariance matrix

$$
\boldsymbol{\Sigma}_{\boldsymbol{\theta}}=\operatorname{diag}\left(\sigma_{\tau}^{2} \boldsymbol{\Sigma}_{a_{x}, \Delta a_{c}, \phi_{x}, \Delta \phi_{c}} \sigma_{\delta \omega_{\mathrm{s}}}^{2} \sigma_{t_{0}}^{2} \sigma_{\Delta \phi_{\mathrm{R}}}^{2}\right),
$$

with channel coefficient covariance matrix $\boldsymbol{\Sigma}_{a_{\mathrm{x}}, \Delta a_{c}, \phi_{\mathrm{x}}, \Delta \phi_{c}}$. The remaining parameters have no prior information $\left(\sigma_{\tau}^{2}=\sigma_{a_{\mathrm{x}}}^{2}=\sigma_{\phi_{\mathrm{x}}}^{2}=\sigma_{\delta \omega_{\mathrm{S}}}^{2}=\sigma_{t_{0}}^{2}=\sigma_{\Delta \phi_{\mathrm{R}}}^{2}=\infty^{6}\right)$.

This improved CRLB helps to quantify the possible precision amelioration of an estimator which is exploiting not only the signal model observation but also prior information on the channel coefficients.

\section{B. Propagation channel statistics}

The covariance matrix $\boldsymbol{\Sigma}_{a_{\mathrm{x}}, \Delta a_{c}, \phi_{\mathrm{x}}, \Delta \phi_{c}}$ is obtained numerically by statistical simulation of ETSI Typical urban (Tux), Rural area (Rax) and Hilly Terrain (HTx) [17] tapped delay line channel models. A random and a frequency flat channel are also considered. The channel impulse response is given by

$$
h(t)=\sum_{p=1}^{P} \beta_{p} \delta\left(t-\left(\tau+\tilde{\tau}_{p}\right)\right),
$$

${ }^{6}$ In numerical simulation $\infty$ is set to $10^{20}$.
TABLE I

RADIO CHANNEL MODEL CHARACTERISTICS

\begin{tabular}{c||r|r}
\hline Channel model & $\tau_{\text {RMS }}$ in $\boldsymbol{\mu s}$ & $B W_{\mathbf{c}}$ in $\mathbf{M H z}$ \\
\hline \hline random & $\infty$ & 0 \\
HTx & 3.0 & 0.33 \\
Tux & 0.5 & 2.00 \\
Rax & 0.1 & 10.00 \\
flat & 0 & $\infty$ \\
\hline
\end{tabular}

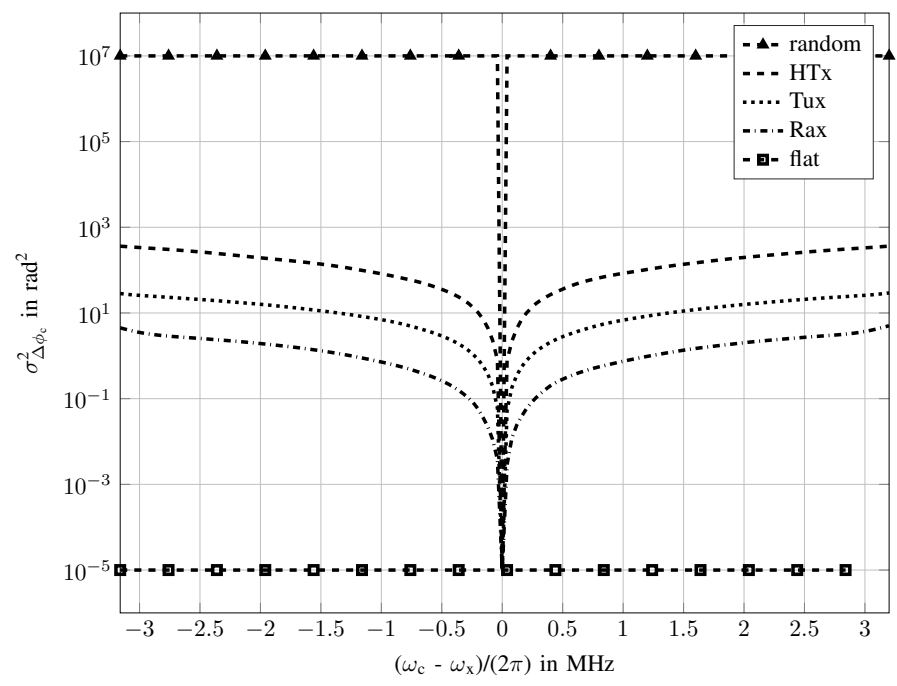

Fig. 3. Phase difference variance for typical ETSI channel models.

with complex path amplitude $\beta_{p}$ for the corresponding path delay offset $\tilde{\tau}_{p}$ w.r.t. the direct path delay $\tau$. The radio channel is assumed time invariant. Root-mean-squared delay $\tau_{\text {RMS }}$ and the empirical coherence bandwidth $B W_{\mathrm{c}}=1 / \tau_{\mathrm{RMS}}$ are given in Table I. For each channel model, $10^{4}$ Rayleigh fading channel realizations are generated in frequency domain. Amplitude and phase differences are calculated as depicted in Fig. 2. Their covariance matrix is assigned according to ${ }^{7}$

$$
\boldsymbol{\Sigma}_{a_{x}, \Delta a_{c}, \phi_{x}, \Delta \phi_{c}}=\operatorname{cov}([\boldsymbol{A} \boldsymbol{\Phi}]) .
$$

Fig. 3 shows the variance of phase differences over the frequency offset from center channel frequency. Variances grow slower for channels with a higher coherence bandwidth.

\section{Numerical CRLBs and analysis}

The delay CRLB is calculated according to (26), with parameter $\boldsymbol{\theta}$, where $\tau \in[0,10 \mathrm{~km}] / c_{0}, \delta \omega_{\mathrm{S}} \in[-1,+1] \mathrm{ppm}$, $t_{0} \in[-100,+100] \mu \mathrm{s}, \Delta \phi_{\mathrm{R}} \in[0,2 \pi]$ uniformly distributed and $\Delta a_{c}, \Delta \phi_{c}$ according to Section IV-B. Results are averaged over $10^{2}$ radio channel realizations.

The bound is given for the sub-GHz ISM band, with a radio frequency $\omega_{\mathrm{RI}}=2 \pi 868 \mathrm{MHz}$. The channel frequency spacing $\omega_{c}=2 \pi 400 \mathrm{kHz}$ yields a maximum unambiguous range ${ }^{8}[10]$

$$
R_{\max }=\pi c_{0} / \omega_{c}=375 \mathrm{~m} .
$$

\footnotetext{
${ }^{7}$ In numerical evaluation, variances for random and flat channel are set to $10^{7}$ and $10^{-} 5$ respectively.

${ }^{8}$ Speed of light $c_{0}=3 \times 10^{8} \mathrm{~m} / \mathrm{s}$.
} 


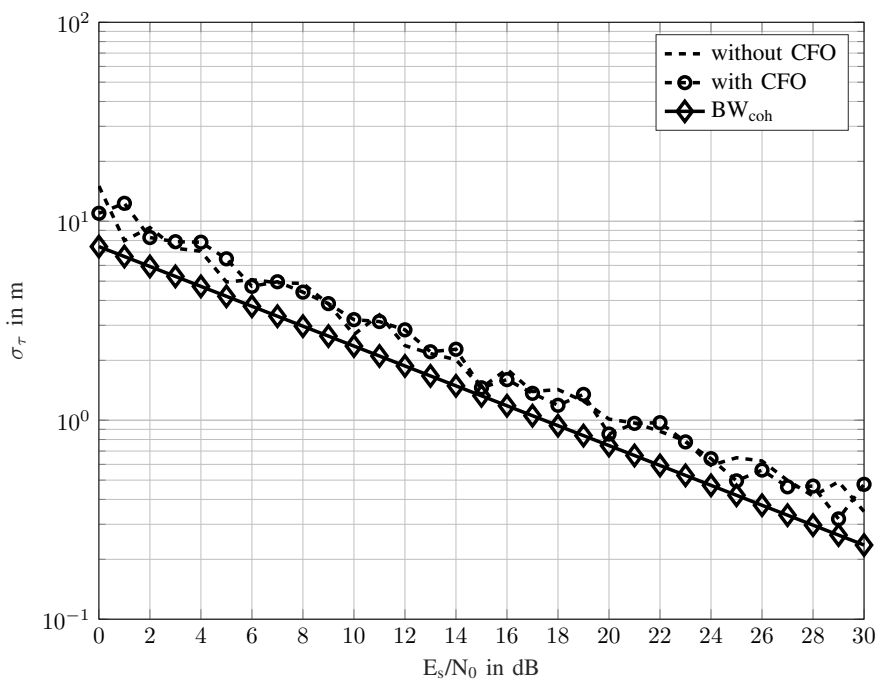

Fig. 4. Delay CRLB without and with carrier frequency offset (CFO).

Range ambiguities beyond $R_{\max }$ can be resolved by single channel ToA [14]. For $C=16$ channels, a coherent bandwidth of $B W_{\text {coh }}=C \omega_{c} /(2 \pi)=6.4 \mathrm{MHz}$ is covered, which exhausts the license-free ISM band. The range resolution is given by [10]

$$
\Delta R=\pi c_{0} /\left(C \omega_{c}\right) \approx 24 \mathrm{~m} .
$$

The narrowband waveform $s_{0}(t)$ is given by a Hanning pulse with duration $T_{\mathrm{sym}}=1 \mathrm{~ms}$ and hence with a LPWAN radio device typical bandwidth of $B W_{\text {sym }}=1 \mathrm{kHz}$. Zero samples for a duration of $T_{\mathrm{z}}=100 \mu \mathrm{s}$ are added before and after the pulse to fulfill the condition of (9). In order to respect the Shannon criteria when $s_{0}(t)$ undergoes CFO, a simulation sampling frequency $\omega_{\mathrm{SI}}=2 \pi \cdot 16 \mathrm{kHz}$ is chosen.

CFO influence: Fig. 4 shows the delay CRLB for the frequency flat channel, for the signal model without and with CFO. Delay estimation in the two-way signaling scheme is not degraded by unknown CFO. For explanation, the signal phase (16) which regroups CFO, synchronization $t_{0}$, initial phases $\phi_{\mathrm{R}}^{[X]}$ and delay $\tau$ terms is considered. It can be seen, that when neglecting the $\mathrm{CFO}$ factor $\left(1+\delta \omega_{\mathrm{S}}\right)$ on the delay parameter, the latter can be eliminated from the other parameters by computing the phase difference $\phi_{\mathrm{F}, c}^{\left[T_{1}, R_{2}\right]}-\phi_{\mathrm{F}, c}^{\left[T_{2}, R_{1}\right]}$. This proves, that CFO estimation can be separated from delay estimation and hence there is no mutual degradation in two-way ranging.

Delay precision: The numerically calculated delay CRLBs for the channel models from Table I are given in Fig. 5. Precision in the frequency flat channel approaches the analytical coherent multi-channel bound $\left(\sigma_{\tau} \approx 1 \mathrm{~m}\right.$ at $\left.\frac{E_{\mathrm{s}}}{N_{0}}=20 \mathrm{~dB}\right)$. For the ETSI channel models, precision degrades with growing RMS delay. For technical relevant $S N R s$, the bounds are $\frac{E_{\mathrm{s}}}{N_{0}}$ independent, showing that the propagation channel and not the system setup limits precision. The precision in the random channel, where no prior on the channel coefficients is available, matches the Non-coherent multi-channel bound ( $\sigma_{\tau, \text { non-coh }} \approx$ $1 \mathrm{~km}$ at $20 \mathrm{~dB}$ ) reported by [16]. For the Typical urban (Tux)

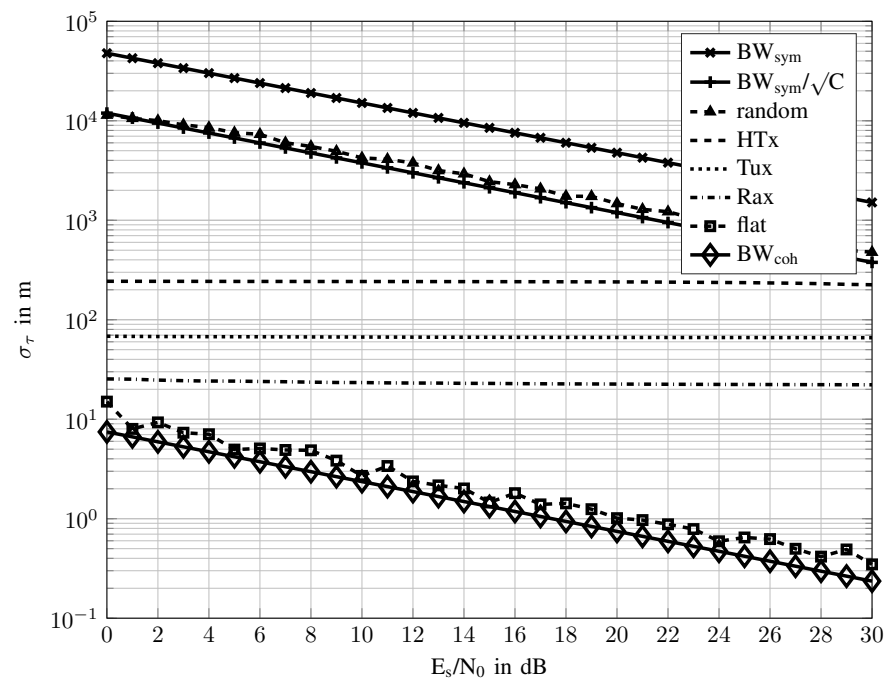

Fig. 5. Numerical delay CRLB for channel models from Table I and analytical CRLBs from Section III-B and $B W_{\text {coh }}=C \omega_{c} /(2 \pi)=6.4 \mathrm{MHz}$.

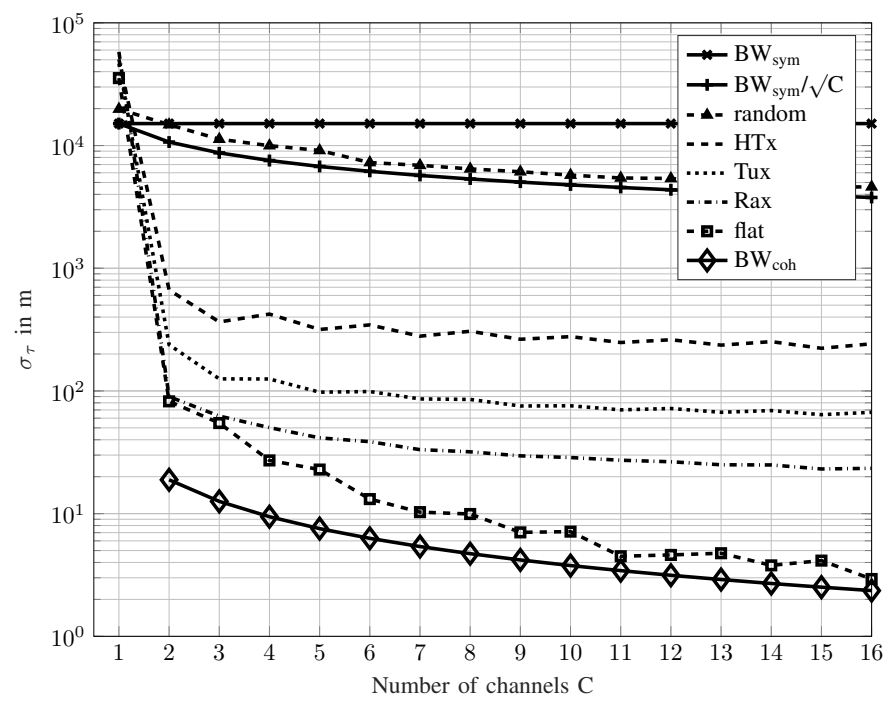

Fig. 6. Delay CRLB as function of bandwidth $B W_{\text {coh }}$ at $\frac{E_{\mathrm{s}}}{N_{0}}=10 \mathrm{~dB}$.

channel, attainable multi-channel ranging precision is $\sigma_{\tau} \leq$ $100 \mathrm{~m}$ at $20 \mathrm{~dB}$, compared to $\sigma_{\tau, \text { single }} \approx \sqrt{C} \sigma_{\tau, \text { non-coh }} \approx 4 \mathrm{~km}$ for a single channel.

Energy consumption - ranging precision trade-off: Fig. 6 shows precision as function of the number of channels $C$. The main precision improvement is observed when using two channels instead of a single channel, where phase information cannot be used. Further improvement for $C>2$, is approximately proportional to $1 / C$ and hence much less significant. Depending on the required range resolution (31), ranging precision and the given radio channel model, reducing the number of channels $C$ is a viable manner to decrease the number of exchanged packets and hence the required energy, which is crucial in the LPWAN context. 


\section{CONCLUSION}

The multi-channel signal model for two-way ranging with $\mathrm{CFO}$ has been extended to include multipath. Analytical expressions for the Jacobian matrix are derived. Multipath statistics are calculated numerically for typical ETSI channel models. These statistics are used, in form of prior information, to give the CRLB on range estimation by numerical evaluation. It is shown, that CFO has no impact on the CRLB bound due to the two-way ranging protocol. Multipath degrades the ranging precision and the main precision gain is achieved, when the coherent multi-channel bandwidth lies within the radio channel coherence bandwidth. The proposed multi-channel approach enables precise ranging for LPWAN radio devices.

Further work should relax the strong hypothesis of constant CFO during the long multi-channel two-way exchange. Therefore, the CRLB study and implementation can be extended to a differential approach with multiple nodes. Moreover, in order to reduce the impact of multipath, the proposed method can be combined with AoA estimation and beamforming to provide further accuracy improvements for LPWAN localization.

\section{APPENDIX \\ JACOBIAN MATRIX}

The Jacobian matrix $J_{\boldsymbol{R}, n} \in \mathbb{R}^{4 C \times(2 C+4)}$ of $\boldsymbol{R}$ is defined as follows

$$
\boldsymbol{J}_{\boldsymbol{R}, n}=\left(\begin{array}{c}
\tilde{\boldsymbol{J}}_{\boldsymbol{R}, n}^{\left[T_{1}, R_{2}\right]} \\
\tilde{\boldsymbol{J}}_{\boldsymbol{R}, n}^{\left[T_{2}, R_{1}\right]}
\end{array}\right) \text { with } \quad \tilde{\boldsymbol{J}}_{\boldsymbol{R}, n}^{[X]}(p, q)=\left(\frac{\partial \boldsymbol{r}_{\mathrm{F}, p}^{[X]}[n]}{\partial \theta_{q}}\right),
$$

with $p \in[0,2 C-1]$ and $q \in[0,2 C+3]$. Partial derivatives for (8) are given as follows. For $\theta_{q} \in\left\{\tau, \delta \omega_{\mathrm{S}}, t_{0}\right\}$

$$
\frac{\partial r_{\mathrm{F}, c}^{[X]}}{\partial \theta_{q}}=a_{c} e^{j \phi_{c}}\left[\frac{\partial s_{0} \partial A_{c}^{[X]}}{\partial A_{c}^{[X]} \partial \theta_{q}}+j s_{0} \frac{\partial B^{[X]}}{\partial \theta_{q}}\right] e^{j B_{c}^{[X]}} .
$$

For $\theta_{q} \in\left\{a_{\mathrm{x}}, \phi_{\mathrm{x}}\right\}$

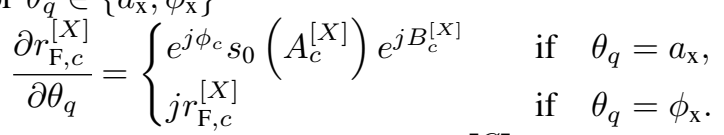

For $\theta_{q} \in\left\{\Delta a_{i}, \Delta \phi_{i}\right\}$ and $i=c \neq\left\lceil\frac{C}{2}\right\rceil-1$

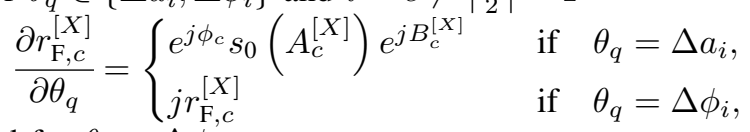

and for $\theta_{q}=\Delta \phi_{\mathrm{R}}$

$$
\frac{\partial r_{\mathrm{F}, c}^{[X]}}{\partial \theta_{q}}=j r_{\mathrm{F}, c}^{[X]} \quad \text { if } \quad[X]=\left[T_{2}, R_{1}\right] .
$$

All other terms are zero. Auxiliary derivatives are given by ${ }^{9}$

$$
\begin{aligned}
& \frac{\partial A_{c}^{[X]}}{\partial \tau}=-\frac{T_{\mathrm{F}}^{[X]}}{T_{\mathrm{SI}}}\left\{\begin{array}{l}
\left(1+\delta \omega_{\mathrm{S}}\right), \\
1,
\end{array}\right. \\
& \frac{\partial B_{c}^{[X]}}{\partial \tau}=\frac{\partial \phi_{\mathrm{F}, c}^{[X]}}{\partial \tau}=-\left(\omega_{c}+\omega_{\mathrm{RI}}\right)\left\{\begin{array}{l}
1, \\
\left(1+\delta \omega_{\mathrm{S}}\right),
\end{array}\right. \\
& \frac{\partial A_{c}^{[X]}}{\partial t_{0}}=-T_{\mathrm{F}}^{[X]} \frac{\partial n_{\mathrm{F}, c}^{[X]}}{\partial t_{0}}=\frac{T_{\mathrm{F}}^{[X]}}{T_{\mathrm{SI}}} \cdot\left\{\begin{array}{l}
-1, \\
\frac{1}{1+\delta \omega_{\mathrm{s}}},
\end{array}\right.
\end{aligned}
$$

${ }^{9}$ Upper choice if $[X]=\left[T_{1}, R_{2}\right]$, lower choice if $[X]=\left[T_{2}, R_{1}\right]$.

$$
\begin{aligned}
& \frac{\partial B_{c}^{[X]}}{\partial t_{0}}=\frac{\partial \phi_{\mathrm{F}, c}^{[X]}}{\partial t_{0}}=\left\{\begin{array}{l}
\frac{-\omega_{c}+\omega_{\mathrm{RI} I} \delta \omega_{\mathrm{S}}}{1+\delta \omega_{\mathrm{S}}} \\
\omega_{c},
\end{array}\right. \\
& \frac{\partial A_{c}^{[X]}}{\partial \delta \omega_{\mathrm{S}}}=\left\{\begin{array}{l}
\frac{-T_{\mathrm{SI}}}{\left(1+\delta \omega_{\mathrm{S}}\right)^{2}}\left(n-n_{\mathrm{F}, c}^{[X]}\right)-\frac{T_{\mathrm{F}}^{[X]}}{T_{\mathrm{SI}}}\left(\tau+l_{c}^{[X]} N_{\mathrm{S}} T_{\mathrm{SI}}\right) \\
T_{\mathrm{SI}}\left(n-n_{\mathrm{F}, c}^{[X]}\right)-\frac{T_{\mathrm{F}}^{[X]}}{T_{\mathrm{SI}}}\left(\frac{t_{0}-l_{c}^{[X]} N_{\mathrm{S}} T_{\mathrm{SI}}}{\left(1+\delta \omega_{\mathrm{S}}\right)^{2}}\right)
\end{array}\right. \\
& \frac{\partial B_{c}^{[X]}}{\partial \delta \omega_{\mathrm{S}}}=n \frac{\partial k_{\mathrm{F}, c}^{[X]}}{\partial \delta \omega_{\mathrm{S}}}+\frac{\partial \phi_{\mathrm{F}, c}^{[X]}}{\partial \delta \omega_{\mathrm{S}}}, \\
& \frac{\partial k_{\mathrm{F}, c}^{[X]}}{\partial \delta \omega_{\mathrm{S}}}=T_{\mathrm{SI}}\left(\omega_{c}+\omega_{\mathrm{RI}}\right)\left\{\begin{array}{l}
-\frac{1}{\left(1+\delta \omega_{\mathrm{S}}\right)^{2}} \\
1,
\end{array}\right. \\
& \frac{\partial \phi_{\mathrm{F}, c}^{[X]}}{\partial \delta \omega_{\mathrm{S}}}=l_{c}^{[X]} N_{\mathrm{S}} \frac{\partial k_{\mathrm{F}, c}^{[X]}}{\partial \delta \omega_{\mathrm{S}}}+\left(\omega_{c}+\omega_{\mathrm{RI}}\right)\left\{\begin{array}{l}
\frac{t_{0}}{\left(1+\delta \omega_{\mathrm{S}}\right)^{2}} \\
-\tau
\end{array}\right.
\end{aligned}
$$

\section{REFERENCES}

[1] U. Raza, P. Kulkarni, and M. Sooriyabandara, "Low Power Wide Area Networks: An Overview," IEEE Communications Surveys Tutorials, vol. 19, no. 2, pp. 855-873, 2017.

[2] Semtech, "SX1276/77/78/79 - $137 \mathrm{MHz}$ to $1020 \mathrm{MHz}$ Low Power Long Range Transceiver," 2016.

[3] Texas Instruments, "CC1125 Ultra-High Performance RF Narrowband Transceiver,"

[4] D. Lachartre, F. Dehmas, C. Bernier, C. Fourtet, L. Ouvry, F. Lepin, E. Mercier, S. Hamard, L. Zirphile, S. Thuries, and F. Chaix, "7.5 A TCXO-less $100 \mathrm{~Hz}$-minimum-bandwidth transceiver for ultra-narrowband sub-GHz IoT cellular networks," in 2017 IEEE International SolidState Circuits Conference (ISSCC), Feb 2017, pp. 134-135.

[5] R. Ratasuk, N. Mangalvedhe, Y. Zhang, M. Robert, and J. P. Koskinen, "Overview of narrowband IoT in LTE Rel-13," in 2016 IEEE Conference on Standards for Communications and Networking (CSCN), Oct 2016, pp. 1-7.

[6] N. Patwari, J. N. Ash, S. Kyperountas, A. O. Hero, R. L. Moses, and N. S. Correal, "Locating the nodes: cooperative localization in wireless sensor networks," IEEE Signal Processing Magazine, vol. 22, no. 4, pp. 54-69, July 2005.

[7] N. Iliev and I. Paprotny, "Review and Comparison of Spatial Localization Methods for Low-Power Wireless Sensor Networks," IEEE Sensors Journal, vol. 15, no. 10, pp. 5971-5987, Oct 2015.

[8] S. Gezici, Z. Tian, G. B. Giannakis, H. Kobayashi, A. F. Molisch, H. V. Poor, and Z. Sahinoglu, "Localization via ultra-wideband radios: a look at positioning aspects for future sensor networks," IEEE Signal Processing Magazine, vol. 22, no. 4, pp. 70-84, July 2005.

[9] S. Lanzisera, D. Zats, and K. S. J. Pister, "Radio Frequency Time-ofFlight Distance Measurement for Low-Cost Wireless Sensor Localization," IEEE Sensors Journal, vol. 11, no. 3, pp. 837-845, March 2011.

[10] M. Skolnik, Radar Handbook, 2nd ed. McGrawHill, 1990.

[11] A. Povalac and J. Sebesta, "Phase difference of arrival distance estimation for RFID tags in frequency domain," in 2011 IEEE International Conference on RFID-Technologies and Applications, Sept 2011, pp. 188-193.

[12] D. Vasisht, S. Kumar, and D. Katabi, "Decimeter-Level Localization with a Single WiFi Access Point," 2016.

[13] M. Pichler, S. Schwarzer, A. Stelzer, and M. Vossiek, "Multi-Channel Distance Measurement With IEEE 802.15.4 (ZigBee) Devices," IEEE Journal of Selected Topics in Signal Processing, vol. 3, no. 5, pp. 845859, Oct 2009.

[14] S. Schwarzer, "Entwicklung eines industriellen Funkortungssystems basierend auf der kohaerenten Kombination von Kommunikationssignalen mit IEEE-802.15.4-Geraeten,' Ph.D. dissertation, Technischen Universitaet Clausthal, 2011.

[15] S. M. Kay, Fundamentals of Statistical Signal Processing: Estimation Theory, 1993.

[16] Y. Karisan, D. Dardari, S. Gezici, A. A. D'Amico, and U. Mengali, "Range Estimation in Multicarrier Systems in the Presence of Interference: Performance Limits and Optimal Signal Design," IEEE Transactions on Wireless Communications, vol. 10, no. 10, pp. 33213331, October 2011.

[17] ETSI, Universal Mobile Telecommunications System (UMTS); Deployment aspects (3GPP TR 25.943 version 9.0.0 Release 9), European Telecommunications Standards Institute (ETSI) Std., 2010. 